\title{
Electroporation-Based ex Vivo Gene Delivery into Dendritic Cells by Anionic Polymer-Coated Versatile Nuclear Localization Signal/pDNA Complex
}

\author{
Takanori Kanazawa, ${ }^{*, a, b}$ Yuki Hoashi, ${ }^{a}$ Hisako Ibaraki, ${ }^{*, a}$ Yuuki Takashima, ${ }^{a}$ and Hiroaki Okada ${ }^{a}$ \\ ${ }^{a}$ School of Pharmacy, Tokyo University of Pharmacy and Life Sciences; 1432-1 Horinouchi, Hachioji, Tokyo 192- \\ 0392, Japan: and ${ }^{b}$ School of Pharmaceutical Sciences, University of Shizuoka; 52-1 Yada, Suruga-ku, Shizuoka \\ 422-8526, Japan. \\ Received July 9, 2021; accepted September 8, 2021
}

In this study, we focused on a nuclear localization signal (NLS)-based versatile peptide vector, designed by us, combined with electroporation (EP) to establish an efficient gene delivery system to non-dividing or slow growing dendritic cells. We determined the intranuclear transport, gene expression, and cell viability in JAWS II mouse dendritic cells transfected with the green fluorescent protein (GFP) expression plasmid DNA alone (naked pEGFP); positive charged complex of NLS derivative STR-CH2SV40H2C, and pEGFP (binary complex); or negative charged complex of the binary complex with a biocompatible polyanion, $\gamma$-polyglutamic acid (ternary complex) combined with or without EP application. Although the binary complex showed higher nuclear transport and GFP expression in the absence of EP than those for naked pEGFP, the combination of EP significantly decreased the cell viability and did not improve the efficiency of compared gene expression. However, in the ternary complex, the intranuclear transport and GFP expression efficiency were significantly higher than those of naked pEGFP and the binary complex when combined with EP, and there was no decrease in cell viability. The results suggest that polyanion-coated ternary complex with EP is useful for non-viral gene delivery system into non-dividing cells for ex vivo gene/cell therapy.

Key words ex vivo gene therapy; nuclear localization signal; electroporation; dendritic cell; nuclear localization signal-based versatile peptide vector

\section{INTRODUCTION}

Dendritic cells (DCs) can induce potent tumor antigenspecific cytotoxic T lymphocytes, which kill tumor cells directly, through the presentation of antigen-derived peptides on MHC class I by the pulsing of antigen-coding plasmid DNA. Therefore, DC-based vaccination is thought to be a promising strategy for cancer immunotherapy. The first DC-associated vaccine, Sipuleucel-T (Provenge ${ }^{\circledR}$ ) for the treatment of minimally symptomatic, hormone refractory prostate cancer was approved by the U.S. Food and Drug Administration (FDA) in 2010. ${ }^{1,2)}$ However, many clinical studies focused on DC-based vaccination suggest insufficient therapeutic benefits. ${ }^{3,4)}$

To ensure the efficient generation of DCs, high antigen expression by delivering a DNA encoding antigen into the nucleus of DCs is needed. However, intranuclear delivery is a formidable barrier for gene delivery into post-mitotic, non- or slow-dividing, and quiescent cells, such as DCs. ${ }^{5)}$ Hence, there is need for safe and efficient gene vectors for clinical application. Two types of gene vectors are used for gene delivery: viral vectors and non-viral vectors. Because viral vectors use the infection mechanism of a virus, their gene transfer efficiency is high. Adeno-associated viral vectors are currently used for clinical applications. However, as viral vectors may be toxic or immunogenic, non-viral vectors with excellent safety need to be developed. Non-viral vectors, including cationic polymers, have several advantages, including non-immunogenicity and low acute toxicity, and have the flexibility for designing a vehicle with well-defined structural and chemical properties to yield mass production.
The nuclear localization signal (NLS), which is recognized by nuclear transport proteins of the importin family, ${ }^{6}$ could overcome the nuclear membrane barrier and promote nuclear translocation. Nuclear delivery is one of the biggest barriers to gene therapy, which may be overcome by the use of NLS. Among NLS sequences, the most extensively studied is the SV40 large-T antigen, especially its shortest oligopeptide, Pro-Lys-Lys-Lys-Arg-Lys-Val. ${ }^{7)}$ However, the NLS hardly enhances gene expression efficiency because it cannot promote DNA packaging-unpacking, cell binding, internalization, and endosomal escape. Therefore, efficient gene delivery using NLS peptides has several requirements, including cellular uptake, endosomal escape, stable plasmid DNA (pDNA) condensation in an extracellular environment, and decondensation in a nuclear environment.

We previously developed STR-CH2SV40H2C, which contains stearic acid (STR), histidine (H), and cysteine (C); it is appropriate for the delivery of therapeutic genes. STR$\mathrm{CH} 2 \mathrm{SV} 40 \mathrm{H} 2 \mathrm{C}$ enhanced transfection efficiency because it facilitated condensation and efficient intracellular and intranuclear delivery of pDNA in DCs and in vivo DNA vaccines, ${ }^{8,9)}$ and efficient release of pDNA in the nucleus by the cleavage of disulfide cross linkages in the intranuclear environment. In contrast, basic peptide vectors with a strong cationic charge have been reported to show cytotoxicity and agglutination owing to their strong electrostatic interaction. One promising approach for overcoming these disadvantages is the formation of a ternary complex coated with anionic polymers, which modify the surface of the vector. Kurosaki et al. have reported that a ternary complex, pDNA/polyethylenimine (PEI) complex 
coated with anionic polymer $\gamma$-polyglutamic acid ( $\gamma$-PGA), shows higher gene expression than the commercial transfection reagent Lipofectin, and it is comparable to a pDNA/PEI complex. This complex does not lead to cytotoxicity and the agglutination of erythrocytes. Moreover, the pDNA/PEI $/ \gamma$-PGA ternary complex was taken up by an energy-dependent process and $\gamma$-PGA specific receptor-mediated pathway. ${ }^{10-12)}$ In addition, previous reports have shown that electroporation is an effective method for in vitro gene transfer by direct delivery into DCs. ${ }^{13,14)}$ We also previously reported the elevation of pDNA nuclear transport, but not intracellular uptake, using electroporation in human DCs and investigated the combination of poly(lactic-co-glycolic acid) (PLGA)/PEI nanospheres and nuclear factor-kappa B p50 analog in the presence of electroporation to further improve gene delivery to DCs. ${ }^{15)}$

In this study, to establish an efficient gene delivery system using slow-dividing DCs, we focused on the combination of an NLS-based versatile peptide vector and electroporation (EP). The physicochemical characterization, cellular uptake, intranuclear transport, gene expression, and cell viability were determined in slow-dividing mouse DCs (JAWS II) transfected with a positively charged complex of NLS derivative STR$\mathrm{CH} 2 \mathrm{SV} 40 \mathrm{H} 2 \mathrm{C}$ and plasmid enhanced green fluorescent protein (pEGFP) (binary complex) or negatively charged complex of the binary complex with a biocompatible polyanion, $\gamma$-PGA (ternary complex), combined with EP application.

\section{MATERIALS AND METHODS}

Materials Plasmid EGFP-N1 (pEGFP; BD Clontech Laboratories, Inc., CA, U.S.A.), was amplified in Escherichia coli $(\mathrm{DH} 5 \alpha)$ and purified using an Endfree Plasmid Maxi kit (Qiagen Sciences, MD, U.S.A.). The concentration of $\mathrm{pEGFP}$ was determined based on UV absorption at $260 \mathrm{~nm}$. YOYO ${ }^{\circledR}-1$ Iodide (Life Technologies Japan Ltd., Tokyo, Japan) was used for fluorescent labeling of pEGFP. The STR-CH2SV40H2C peptides (Stearoyl-CHHPKKKRKVHHC) were synthesized and purified according to our previous paper. ${ }^{9)}$ JAWS II cells were purchased from American Type Culture Collection (WA, U.S.A.). Cell culture medium, minimum essential medium (MEM) alpha, certified fetal bovine serum (FBS), penicillin/streptomycin stock solutions, and $0.25 \%$ Trypsinethylenediaminetetraacetic acid (EDTA) were purchased from Life Technologies Japan Ltd.. Recombinant mouse granulocyte macrophage colony-stimulating factor (GM-CSF; Wako Pure Chemical Corporation, Osaka, Japan) was used to generate immature DCs.

Preparation of Binary and Ternary pDNA Complexes The pDNA/peptide binary complexes were prepared by mixing at $\mathrm{N} / \mathrm{P}$ ratio $10 / 1$ for $24 \mathrm{~h}$ at $20^{\circ} \mathrm{C}$. The $\gamma$-PGA-coated pDNA/peptide complexes (ternary complexes) were prepared by adding $\gamma$-PGA into binary complexes with components at $\mathrm{O} / \mathrm{N} / \mathrm{P}$ ratio $100 / 10 / 1$ for $30 \mathrm{~min}$ at $20^{\circ} \mathrm{C}$. O/N/P ratios were defined as the molar ratio of hydroxyl groups $(\mathrm{O})$ in carboxylic acid of $\gamma$-PGA/amine groups $(\mathrm{N})$ in lysine and arginine of peptide/DNA phosphate groups in pEGFP (P).

Physiochemical Characterization of pDNA Complexes The mean size and zeta potential of the binary or ternary pDNA complexes were determined using a dynamic light scattering (DLS)-700 unit (Otsuka Electronics Co., Ltd., Osaka, Japan) and Zeta Potential/Particle Sizer NICOMPTM 380 ZLS
(Nicomp Particle Size Systems, Santa Barbara, CA, U.S.A.).

In Vitro Transfection into JAWS II Cells During the process of transfection without electroporation, JAWS II cells were harvested by trypsinization, washed with phosphatebuffered saline (PBS), resuspended in MEM-alpha, and seeded into 6-well culture plates $\left(1 \times 10^{6}\right.$ cells/well $)$. After $24 \mathrm{~h}$ of incubation at $37^{\circ} \mathrm{C}$ in MEM-alpha containing $20 \% \mathrm{FBS}$, the cells were rinsed with MEM-alpha without FBS and transfected with YOYO-1-labeled (excitation/emission:491/509 nm) or non-labeled pEGFP complex solution (pEGFP, $1 \mu \mathrm{g}$ ) in each well. During the transfection by electroporation, JAWS II cells were harvested by trypsinization, washed with PBS, and resuspended in MEM-alpha without FBS. We mixed $20 \mu \mathrm{L}$ of JAWS II cell suspension and $80 \mu \mathrm{L}$ of pEGFP complex solution (pEGFP, $1 \mu \mathrm{g}$ ) in each chamber, and electroporation was carried out using the in vitro electroporator CUY21Pro-vitro (Nepa Gene Co., Ltd., Chiba, Japan) at $125 \mathrm{~V}$ with five pulses. Subsequently, the JAWS II cell suspension was seeded into the 6-well culture plates with MEM-alpha containing 20\% FBS for further incubation.

Detection of Cellular Uptake of pEGFP in JAWS II Cells After $4 \mathrm{~h}$ of transfection of YOYO-1-labeled pEGFP complexes, the JAWS II cells were washed and detached by pipetting and resuspended in PBS. YOYO-1 fluorescence intensity in the cells was analyzed using flow cytometry (FACSCanto; Japan BD Co., Ltd., Tokyo, Japan). The transfected cells were detected with subtracting the autofluorescence. We analyzed 30000 events and depicted the YOYO-1 detected cells among all transfected cells as the cellular uptake efficiency (\%).

Determination of Amount of Intranuclear pEGFP in JAWS II Cells After $4 \mathrm{~h}$ of transfection of YOYO-1-labeled pEGFP complexes, the nuclei were collected from JAWS II cells according to our previous papers. ${ }^{9,15)}$ After lysis buffer was added to the cells and incubated for at least $5 \mathrm{~min}$, cell lysate suspension was centrifuged and the nuclei was collected and resuspended in PBS. The number of nuclei was counted nuclei suspension lysate by microscopic observation (Olympus IX71, Olympus Corporation, Tokyo, Japan) with hemocytometer (Sansyo Co., Ltd., Tokyo, Japan), and the fluorescence intensity of YOYO-1-labeled pEGFP in the nuclei was analyzed using a microplate reader at $491 / 509 \mathrm{~nm}$ (Safire Microplate Reader; Tecan) after centrifugation. The amount of YOYO1-labeled pEGFP was calculated using a calibration curve generated with YOYO-1-labeled pEGFP standards. The results are shown as the amount of pEGFP per nucleus.

Detection of GFP Expression in JAWS II Cells After $48 \mathrm{~h}$ of transfection of pEGFP complexes, the GFP fluorescence intensity in the JAWS II cells was analyzed using flow cytometry (FACSCanto; Japan BD Co., Ltd., Tokyo, Japan). We analyzed 30000 events and the GFP-expressing cells among all transfected cells are shown as gene transfection efficiency (\%).

Statistical Analysis The statistical analysis between the mean values of multiple groups was evaluated using ANOVA with Tukey's test using Bell Curve in Microsoft Excel was used for statistical analysis. Statistical significance was set as $p<0.05$.

\section{RESULTS}

Physicochemical Characterization of Binary or Ternary pDNA Complex Table 1 shows the size and surface po- 
tential of binary and ternary complexes. They represent the average value $(n=3)$. The binary complex, which constituted cationic STR-CH2SV40H2C and pEGFP, had a size of $99.7 \mathrm{~nm}$ and zeta potential of $+34.1 \mathrm{mV}$. The ternary complex, which constituted a binary complex coated with $\gamma$-PGA, had a size of $134.5 \mathrm{~nm}$ and negative charge of $-33.5 \mathrm{mV}$.

pEGFP Cellular Uptake and Cell Viability in JAWS Cells Transfected with Binary or Ternary Complexes by Electroporation Figure 1a shows the intracellular uptake efficiency of naked pEGFP, binary complexes, and ternary complexes transfected into JAWS II cells after $4 \mathrm{~h}$, with and without electroporation. Figure 1b shows the fluorescence intensity per cell.

Cellular uptake of pEGFP was hardly observed with naked pEGFP, and binary and ternary complexes showed a higher

Table 1. Mean Diameter and Zeta Potential of Binary or Ternary pEGFP Complex

\begin{tabular}{lcc}
\hline \hline pEGFP complex & Mean diameter $(\mathrm{nm})$ & Zeta potential $(\mathrm{mV})$ \\
\hline Binary complex & 99.7 & +34.1 \\
Ternary complex & 134.5 & -33.5 \\
\hline
\end{tabular}

(a)

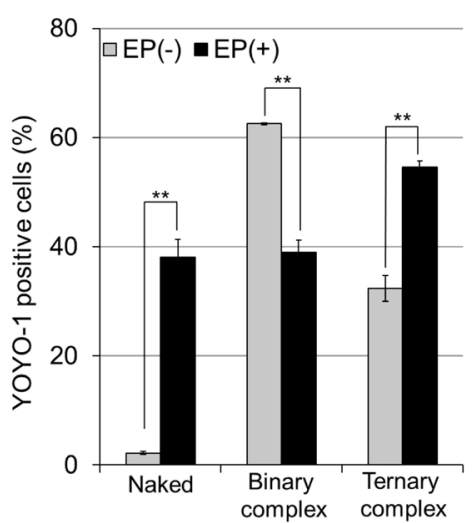

cellular uptake efficiency of pEGFP than naked pEFGP in the absence of electroporation. In particular, the binary complex showed the highest uptake. Next, in the presence of electroporation, the cellular uptake efficiency of pEGFP by the binary complex was significantly lower than that by the nonelectroporated binary complex. This phenomenon indicated that electroporation did not promote uptake by the binary complex. In contrast, the cellular uptake of pEGFP by naked pEGFP and ternary complexes was significantly increased in the presence of electroporation, especially by the ternary complexes, which showed the highest cellular pEGFP uptake.

Figure 2 shows the cytotoxicity of naked pEGFP, binary, and ternary complexes to JWAS II cells. Naked pEGFP and binary complexes showed high cytotoxicity in the presence of electroporation, although no decrease in cell viability was observed in the absence of electroporation. In contrast, the ternary complex, in which the binary complex was coated with $\gamma$-PGA, a polyanion, strongly suppressed the decrease in cell viability in the presence of electroporation.

Effect of Electroporation on the Physicochemical Characterization of Binary or Ternary Complexes Figure 3 shows the mean size and zeta potential of the binary and ter-

(b)

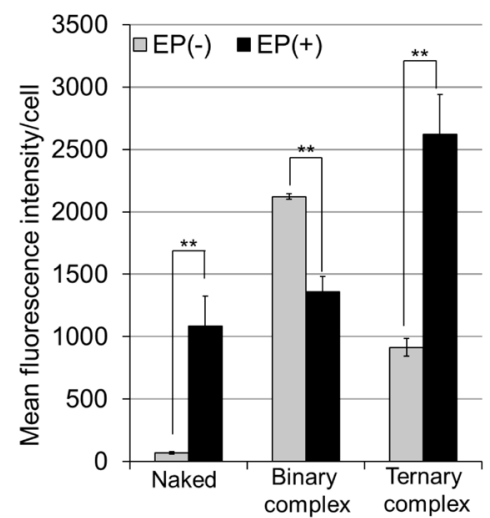

Fig. 1. Cellular Uptake Ability of pDNA in JAWS II Cells Transfected with Naked YOYO-1 pEGFP, Binary, and Ternary Complexes with or without Electroporation: (a) Intracellular Uptake Efficiency (\%); (b) Mean Fluorescence Intensity of YOYO-1 per Cell

Each bar represents the mean \pm standard deviation (S.D.) $(n=3)$. ${ }^{* *} p<0.01$ (ANOVA).

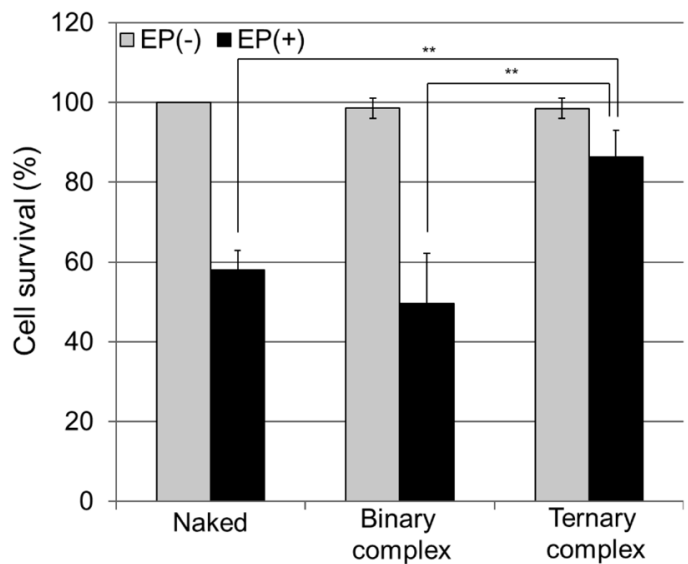

Fig. 2. In Vitro Cytotoxicity in JAWS II Cells Transfected with Naked pEGFP and pEGFP Binary and Ternary Complexes with or without Electroporation

Each bar represents the mean \pm S.D. $(n=3) . * * p<0.01$ (ANOVA).

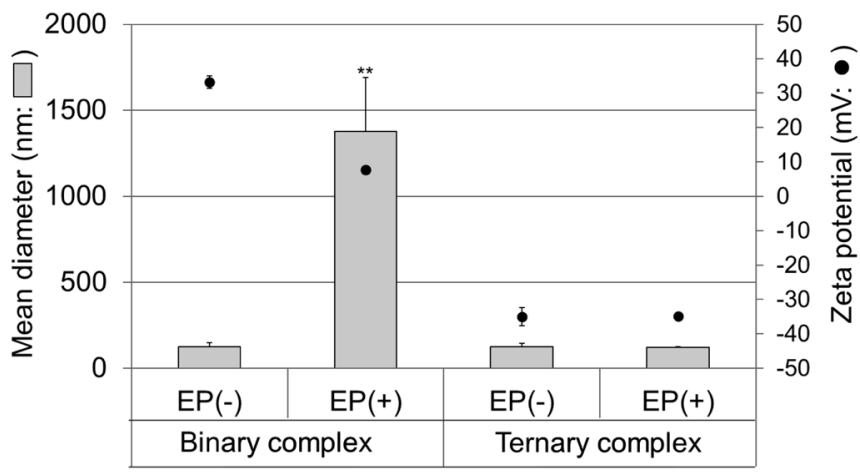

Fig. 3. Mean Diameter $(\mathrm{nm})$ and Zeta Potential $(\mathrm{mV})$ of pEGFP Binary and Ternary Complexes before and after Electroporation (EP)

Each bar represents the mean \pm S.D. $(n=3) . * * p<0.01 v s$. other groups (ANOVA). 
nary complexes before and after electroporation. In the binary complex, electroporation led to an increase in complex size, probably owing to aggregation or deformation. In contrast, ternary complexes coated with polyanions maintained almost the same physical properties after electroporation.

Intranuclear pDNA Amount Figure 4 shows the amount of intranuclear pEGFP in JWAS II cells after $4 \mathrm{~h}$ of transfection with naked pEGFP, binary complex, and ternary complex. The intranuclear amount of naked pEGFP increased by electroporation, whereas intranuclear naked pEGFP was never detected without electroporation. With the ternary complex, a significant increase was observed in the amount of intranuclear pEGFP in the presence of electroporation compared with the other groups. In contrast, the binary complexes showed an increase in the amount of intranuclear pEGFP in the absence of electroporation, whereas a significant decrease in the amount of intranuclear pEFGP was observed in the presence of electroporation.

Gene Expression Efficiency Figure 5 shows that the gene expression efficiency in JWAS II cells after transfection of bi-

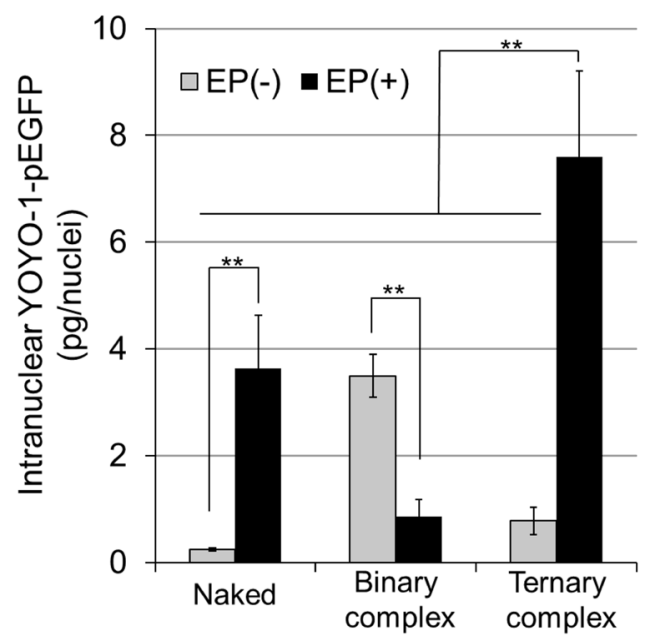

Fig. 4. Intranuclear Amount of YOYO-1 pEGFP in JAWS II Cells Transfected with Naked YOYO-1 pEGFP, Binary and Ternary Complexes with or without Electroporation

Each bar represents the mean \pm S.D. $(n=3) . * * p<0.01$ (ANOVA).

(a)

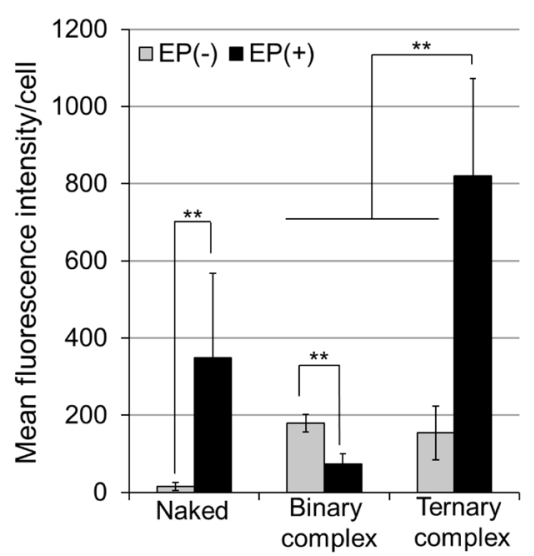

nary and ternary complexes with and without electroporation. In the absence of electroporation, GFP expression efficiency of binary complexes and ternary complexes was significantly higher than that of naked pEFGP. In contrast, with electroporation, the GFP expression efficiency of binary complexes was significantly decreased, whereas that of naked and ternary complexes significantly increased. In particular, the ternary complexes showed the highest GFP expression efficiency with electroporation.

\section{DISCUSSION}

In recent years, cell therapy has been developed as a new treatment for malignant tumors, in which immune-related cells, such as T cells and DCs, collected from patients are activated by ex vivo gene transfer and administered to patients again. However, as these cells are differentiated cells, their nuclear membranes are rarely divided during mitosis. Therefore, the nuclear membrane is the biggest barrier for the development of ex vivo gene/cell therapy. In this study, to develop an efficient ex vivo gene delivery system for DCs based on the non-viral NLS peptide vector, we investigated the enhancement of the gene expression efficiency in DCs of a binary complex consisting of a versatile peptide vector developed by us and pEGFP combined with electroporation, which is a representative physical gene transfer method. In addition, to reduce the toxicity of the positively charged binary complexes, ternary complexes, which constitute binary complexes coated with $\gamma$-PGA, a biodegradable polyanion, were also examined.

First, binary and ternary complexes were prepared and their physical properties were evaluated (Table 1). As a result, the binary complex consisting of a versatile STR-CH2SV40H2C vector and $\mathrm{pEGFP}$ was around $100 \mathrm{~nm}$ and had a zeta potential of around $30 \mathrm{mV}$, which was similar to our previous report.9) The ternary complex constituting the $\gamma$-PGA-coated binary complex had a larger size than the binary complex and a strong negative charge, suggesting that the ternary complex was coated with $\gamma$-PGA. Regarding the $\mathrm{O} / \mathrm{P}$ ratio, the size and zeta-potential of the ternary complexes at various $\mathrm{O} / \mathrm{P}$ ratios $(\gamma$-PGA/pDNA) from 0 to 250 were measured as preliminary experiment (Supplementary Figure). As a result, the zeta-

(b)

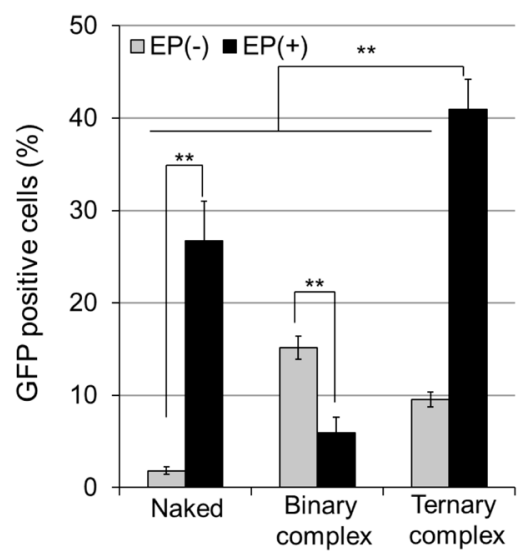

Fig. 5. GFP Expression Efficiency in JAWS II Cells Transfected with Naked pEGFP and pEGFP Binary and Ternary Complexes with or without Electroporation: (a) GFP Positive Cells (\%); (b) Mean Fluorescence Intensity of GFP per Cell

Each bar represents the mean \pm S.D. $(n=3) . * * p<0.01$ (ANOVA). 
potential was almost unchanged at $-30 \mathrm{mV}$ at an $\mathrm{O} / \mathrm{P}$ ratio of above 100, and the size was smallest at $\mathrm{O} / \mathrm{P} 100$, therefore $\mathrm{O} / \mathrm{P}=100 / 1$ were selected in this study. Now we are planning the analysis of these complexes by Cryo-TEM or AFM to understand the physiochemical characterization and uniformity of binary and ternary complexes.

The cellular pEGFP uptake efficiency of both binary and ternary complexes was increased in the absence of EP (Fig. 1). In general, previous reports have shown that basic peptides adhere to cell membranes and increase cellular pDNA uptake because of their positive charge. ${ }^{16-19)}$ Additionally, it has been reported that the modification of fatty acids to basic peptides, such as STR, enhances their cellular uptake. ${ }^{7,16,17)}$ The NLS peptide vector that constitutes the binary complex in this study is SV40, which is rich in basic amino acids, and has undergone STR modification, which may have resulted in a significant increase in cellular pDNA uptake. It has also been shown that $\gamma$-PGA adheres to the cell membrane via specific receptors and is taken up into cells by caveolae-mediated endocytosis. ${ }^{11)}$ Therefore, we speculated that the cellular uptake of our ternary complex coated with $\gamma$-PGA would be enhanced by the same pathway. Furthermore, the cellular pDNA uptake of naked and ternary complexes combined with electroporation significantly increased cellular intake than the absence of electroporation. In contrast, the cellular pDNA uptake of binary complexes was significantly decreased because of electroporation (Fig. 1). In addition, although there was no decrease in cell viability in the absence of electroporation in cells transfected with naked pEFGP and both complexes, the combination of electroporation resulted in high cytotoxicity by the naked and binary complexes (Fig. 2). In contrast, the ternary complex coated with polyanion $\gamma$-PGA suppressed the decrease in cell viability when combined with electroporation (Fig. 2). This low toxicity is thought to contribute to high transfection efficiency. In the case of the binary complex combined with electroporation, either large aggregates or deformation was observed and the surface potential was greatly reduced. However, in the case of the ternary complex, the same physical properties were observed before and after electroporation (Fig. 3). This finding suggests that the binary complex, which has highly cationic properties, is susceptible to the effects of electroporation with a strong electric field. In addition, the interaction of STR-CH2SV40H2C with the cell membrane was significantly altered by the change in the physical properties of STR-CH2SV40H2C, which may have resulted in the inhibition of uptake and induction of cytotoxicity. In contrast, the physical properties of the negatively charged ternary complexes coated with polyanions were less affected by electroporation, showing that the enhancement of cellular effect by electroporation can be obtained while forming a stable complex. Therefore, it was suggested that $\gamma$-PGA coating not only improved the decrease of cell viability by electroporation but also greatly contributed to the enhancement of cellular uptake by electroporation with stable physical properties.

Then, the amount of intranuclear pEGFP in JAWS II cells was examined (Fig. 4). In naked pEGFP transfected cells, no intranuclear pEGFP was observed without electroporation, whereas an increase in the amount of intranuclear pEGFP was observed with electroporation. In contrast, the binary complexes showed high nuclear migration in the absence of electroporation, but the nuclear migration was markedly reduced with electroporation. This may be owing to the disintegration of the binary complex by electroporation and marked decrease in cellular uptake. The ternary complexes showed intranuclear pEGFP even in the absence of electroporation, indicating that the intracellular uptake by $\gamma$-PGA and intranuclear transfer by NLS peptide are possible. Furthermore, a significant increase in the amount of intranuclear pEGFP was observed with the ternary complex in the presence of electroporation compared with the other groups. This suggests that the ternary complexes were delivered to the cytoplasm as well as the nucleus by electroporation, with the formation of a stable complex.

Finally, gene expression efficiency was examined. The GFP expression efficiency of naked and ternary complexes was significantly increased by electroporation and was the highest, whereas that of binary complexes was significantly decreased (Fig. 5). This result was strongly correlated with the amount of intranuclear pDNA, suggesting that pDNA transferred into the nucleus is expressed in the nucleus of DCs. This result strongly correlated with the amount of nuclear transfer, suggesting that pDNA transferred into the nucleus is important for gene expression in DCs. In addition, electroporation not only improved the intracellular uptake of naked pEGFP but also the nuclear transfer and gene transfer efficiency, indicating that electroporation not only aids intracellular transfer but also nuclear transfer ex vivo.

These results indicate that electroporation not only improves the cellular uptake of naked pEGFP but also the nuclear transfer and gene transfer efficiency. A significant improvement was observed in ternary complexes concerning the efficiency of intracellular uptake, nuclear transfer, and gene expression in the presence of electroporation. This may be owing to the additive effect of electroporation on cellular uptake and nuclear translocation, as well as cellular uptake induced by $\gamma$-PGA and intracellular dynamics, including nuclear transfer, by our versatile NLS peptide vector constituting a ternary complex. We are planning to evaluate their gene expression efficacy using other dendritic cell lines and primary cells such as Bone marrow-derived dendritic cell.

\section{CONCLUSION}

In this study, a ternary complex comprising pEGFP, STR$\mathrm{CH} 2 \mathrm{SV} 40 \mathrm{H} 2 \mathrm{C}$ as a versatile NLS gene vector, and $\gamma$-PGA as a polyanion was used for gene delivery by electroporation. The efficiency of gene expression was remarkably improved, whereas cytotoxicity was suppressed, in DCs, to which it has been difficult to achieve efficient gene delivery. Currently, there are not enough safe and effective methods of gene delivery to non-dividing DCs using non-viral vectors. Therefore, efficient gene delivery to DCs, as shown in this study, is expected to aid the future development of DC vaccines.

Acknowledgments We would like to thank Nepa Gene Co., Ltd. (Chiba, Japan) for kindly supplying the in vitro electroporator. We also appreciate the technical assistance provided by Mr. Kazuya Nigoro, Ms. Asuna Shibano, and Ms. Kana Sogabe.

Author Contributions Takanori Kanazawa: Conceptualization, Methodology, Validation, Formal analysis, Investigation, Data curation, Writing - original draft preparation, 
Visualization, Project administration. Yuki Hoashi: Methodology, Validation, Formal analysis, Data curation, Visualization. Hisako Ibaraki: Methodology, Formal analysis, Data curation. Yuuki Takashima: Writing-review and editing. Hiroaki Okada: Writing - review and editing, Supervision.

Conflict of Interest The authors declare no conflict of interest.

Supplementary Materials The online version of this article contains supplementary materials.

\section{REFERENCES}

1) Gardner TA, Elzey BD, Hahn NM. Sipuleucel-T (Provenge) autologous vaccine approved for treatment of men with asymptomatic or minimally symptomatic castrate-resistant metastatic prostate cancer. Hum. Vaccin. Immunother., 8, 534-539 (2012). https://doi. org $/ 10.4161 /$ hv.19795

2) Wooster AL, Girgis LH, Brazeale H, Anderson TS, Wood LM, Lowe DB. Dendritic cell vaccine therapy for colorectal cancer. Pharmacol. Res., 164, 105374 (2021). https://doi.org/10.1016/j.phrs. 2020.105374

3) Banchereau J, Palucka AK. Dendritic cells as therapeutic vaccines against cancer. Nat. Rev. Immunol., 5, 296-306 (2005). https://doi. org/10.1038/nri1592.

4) Miki M, Nagaoka K, Harada M, Hayashi T, Jinguji H, Kato Y, Maekawa R. Combination therapy with dendritic cell vaccine and IL-2 encapsulating polymeric micelles enhances intra-tumoral accumulation of antigen-specific CTLs. Int. Immunopharmacol., 23, 499-504 (2014). https://doi.org/10.1016/j.intimp.2014.09.025.

5) Wiethoff CM, Middaugh CR. Barriers to nonviral gene delivery. J. Pharm. Sci., 92, 203-217 (2003). https://doi.org/10.1002/jps.10286.

6) Pouton CW, Wagstaff KM, Roth DM, Moseley GW, Jans DA. Targeted delivery to the nucleus. Adv. Drug Deliv. Rev., 59, 698-717 (2007). https://doi.org/10.1016/j.addr.2007.06.010.

7) Wang HY, Chen JX, Sun YX, Deng JZ, Li C, Zhang XZ, Zhuo RX. Construction of cell penetrating peptide vectors with $\mathrm{N}$-terminal stearylated nuclear localization signal for targeted delivery of DNA into the cell nuclei. J. Control. Release, 155, 26-33 (2011). https://doi.org/10.1016/j.jconrel.2010.12.009.

8) Kanazawa T, Tamura T, Yamazaki M, Takashima Y, Okada H. Needle-free intravaginal DNA vaccination using a stearoyl oligopeptide carrier promotes local gene expression and immune responses. Int. J. Pharm., 447, 70-74 (2013). https://doi.org/10.1016/j. ijpharm.2013.02.018.

9) Kanazawa T, Yamazaki M, Fukuda T, Takashima Y, Okada H. Versatile nuclear localization signal-based oligopeptide as a gene vector. Biol. Pharm. Bull., 38, 559-565 (2015). https://doi.org/ 10.1248/bpb.b14-00706.

10) Kanda $K$, Kodama $Y$, Kurosaki $T$, Imamura M, Nakagawa $H$, Muro T, Higuchi N, Nakamura T, Kitahara T, Honda M, Sasaki H. Ternary complex of plasmid DNA with protamine and $\gamma$-polyglutamic acid for biocompatible gene delivery system. Biol. Pharm. Bull., 36, 1794-1799 (2013). https://doi.org/10.1248/bpb.b13-00479.

11) Kurosaki $T$, Kitahara $T$, Fumoto $S$, Nishida $K$, Nakamura J, Niidome T, Kodama Y, Nakagawa H, To H, Sasaki H. Ternary complexes of pDNA, polyethylenimine, and gamma-polyglutamic acid for gene delivery systems. Biomaterials, 30, 2846-2853 (2009). https:// doi.org/10.1016/j.biomaterials.2009.01.055.

12) Kurosaki T, Kitahara T, Kawakami S, Higuchi Y, Yamaguchi A, Nakagawa H, Kodama Y, Hamamoto T, Hashida M, Sasaki H. Gamma-polyglutamic acid-coated vectors for effective and safe gene therapy. J. Control. Release, 142, 404-410 (2010). https://doi.org/ 10.1016/j.jconrel.2009.11.010.

13) Artusio E, Hathaway B, Stanson J, Whiteside TL. Transfection of human monocyte-derived dendritic cells with native tumor DNA induces antigen specific T-cell responses in vitro. Cancer Biol. Ther., 5, 1624-1631 (2006). https://doi.org/10.4161/cbt.5.12.3353.

14) Simon GG, Hu Y, Khan AM, Zhou J, Salmon J, Chikhlikar PR, Jung KO, Marques ET, August JT. Dendritic cell mediated delivery of plasmid DNA encoding LAMP/HIV-1 Gag fusion immunogen enhances $\mathrm{T}$ cell epitope responses in HLA DR4 transgenic mice. PLoS ONE, 5, e8574 (2010). https://doi.org/10.1371/journal. pone.0008574

15) Kanazawa T, Takashima $Y$, Murakoshi M, Nakai Y, Okada H. Enhancement of gene transfection into human dendritic cells using cationic PLGA nanospheres with a synthesized nuclear localization signal. Int. J. Pharm., 379, 187-195 (2009). https://doi.org/10.1016/j. ijpharm.2009.06.015.

16) Futaki S, Ohashi W, Suzuki T, Niwa M, Tanaka S, Ueda K, Harashima H, Sugiura Y. Stearylated arginine-rich peptides: a new class of transfection systems. Bioconjug. Chem., 12, 1005-1011 (2001). https://doi.org/10.1021/bc0155081

17) Khalil IA, Futaki S, Niwa M, Baba Y, Kaji N, Kamiya H, Harashima $\mathrm{H}$. Mechanism of improved gene transfer by the N-terminal stearylation of octaarginine: enhanced cellular association by hydrophobic core formation. Gene Ther., 11, 636-644 (2004). https:// doi.org/10.1038/sj.gt.3302128

18) Nakamura Y, Kogure K, Futaki S, Harashima H. Octaargininemodified multifunctional envelope-type nano device for siRNA. J. Control. Release, 119, 360-367 (2007). https://doi.org/10.1016/j. jconrel.2007.03.010

19) Hashida H, Miyamoto M, Cho Y, Hida Y, Kato K, Kurokawa T, Okushiba S, Kondo S, Dosaka-Akita H, Katoh H. Fusion of HIV-1 Tat protein transduction domain to poly-lysine as a new DNA delivery tool. Br. J. Cancer, 90, 1252-1258 (2004). https://doi.org/ 10.1038/sj.bjc.6601680. 